\title{
Sarcoidosis al descubierto: Lo que deberíamos informar en las imágenes torácicas
}

\section{Sarcoidosis Uncovered: What we should Report in Thoracic Image}

\author{
Javier Mauricio Martínez Martínez ${ }^{1 \odot} \quad$ Karen Margarita Palacio Vargas ${ }^{1 \odot} \quad$ María del Mar Astorga Lahoz ${ }^{1}$ \\ Manuel Conde Blanco ${ }^{1 \odot}$ María Cecilia Ferrario ${ }^{1}$ Pablo Matías Aguirre ${ }^{1 \odot}$ \\ ${ }^{1}$ Servicio de Diagnóstico por Imágenes, Hospital Universitario \\ Austral, Presidente Derqui, Pilar, Buenos Aires, Argentina \\ Rev Argent Radiol 2019;83:77-86. \\ Address for correspondence Javier Mauricio Martínez Martínez, MD, \\ Servicio de Diagnóstico por Imágenes, Hospital Universitario Austral, \\ Presidente Derqui, Pilar, CP 1629, Buenos Aires, Argentina \\ (e-mail: jamartin@cas.austral.edu.ar).
}

\section{Resumen}

Palabras clave

- sarcoidosis

- radiografía

- tomografía computada

- tomografía por emisión de positrones

- resonancia magnética
La sarcoidosis es una enfermedad granulomatosa no caseificante, multisistémica, de causa desconocida, que compromete al pulmón y a los ganglios linfáticos mediastinales entre el 90 y el $95 \%$ de los casos. También puede afectar otros órganos, como las glándulas salivales, piel, ojos, hígado, bazo, corazón, huesos y sistema nervioso central. La sarcoidosis tiene una baja prevalencia en Latinoamérica y es subdiagnosticada debido a la alta frecuencia de otros trastornos similares, como tuberculosis, lepra y micosis profundas.

El diagnóstico presuntivo se establece con hallazgos imagenológicos característicos dentro de un contexto clínico apropiado y se confirma con la evidencia histológica de granulomas no caseificantes de células epiteliales, en ausencia de otras etiologías. Los hallazgos torácicos incluyen la afectación pulmonar, ganglionar y bronquial, los cuales son detectados a través de la radiografía (Rx) y tomografía computada (TC) de tórax, siendo esa última más sensible y específica.

En este artículo, resaltamos la importancia de reconocer los patrones de presentación típicos y atípicos de la sarcoidosis en Rx y TC, así como la relevancia de las imágenes torácicas como elemento clave en el algoritmo diagnóstico de esa patología. También describimos la utilidad de la resonancia magnética (RM), como método adicional para el diagnóstico en casos de afectación cardíaca y el papel de la tomografía por emisión de positrones (PET-CT) en el seguimiento terapéutico.

\section{Introducción}

La sarcoidosis es una enfermedad granulomatosa no caseificante, multisistémica, de causa desconocida que en la mayoría de los casos compromete al pulmón y a los ganglios linfáticos mediastinales. También puede afectar otros órganos, como las glándulas salivales, la piel, los ojos, el hígado, el bazo, el corazón, los huesos y el sistema nervioso central. El compromiso torácico representa la principal causa de morbilidad y mortalidad en pacientes con dicha patología.

\footnotetext{
(D)Javier Mauricio Martínez's ORCID is https://orcid.org/0000-0003-1610-9708.

(1) Karen Margarita Palacio Vargas's ORCID is https://orcid.org/0000-0002-9182-5045.

(1) Manuel Conde Blanco's ORCID is https://orcid.org/0000-0003-1463-8745.

(1) Pablo Matías Aguirre's ORCID is https://orcid.org/0000-0003-1021-2528.
}

received

March 9, 2018

accepted

May 6, 2019
DOI https://doi.org/

10.1055/s-0039-1692153.

ISSN 1852-9992.
Copyright $\odot$ 2019, Sociedad Argentina de Radiología. Publicado por Thieme Revinter Publicações Ltda., Rio de Janeiro, Brazil. Todos los derechos reservados.
License terms

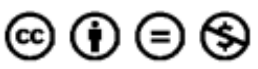




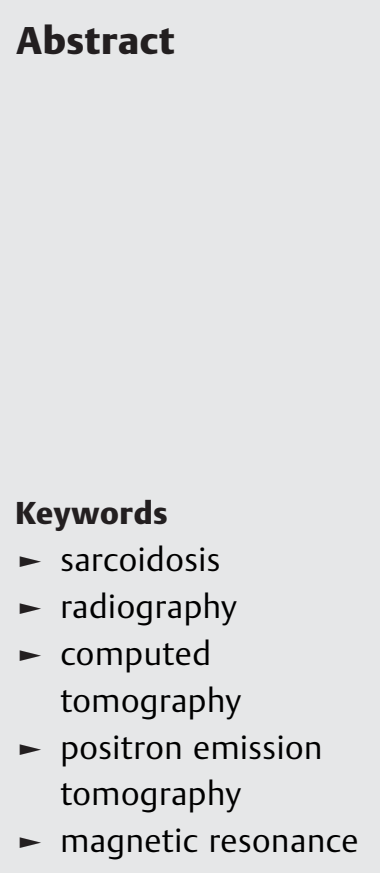

Sarcoidosis is a non-caseating granulomatous, multisystemic disease of unknown cause that involves the lung and mediastinal lymph nodes in $90-95 \%$ of cases. It can also affect other organs such as the salivary glands, skin, eyes, liver, spleen, heart, bones and the central nervous system. Sarcoidosis has a low prevalence in Latin America and it is underdiagnosed due to the high frequency of other similar disorders such as tuberculosis, leprosy and deep mycosis.

The presumptive diagnosis is established based on characteristic imaging findings within an appropriate clinical setting and is confirmed by histological evidence of noncaseating epithelioid cell granulomas, in the absence of other etiologies.

Thoracic imaging findings include pulmonary, nodal and bronchial involvement, which are detected on chest radiography (CXR) and computed tomography (CT), this last one having a higher sensitivity and specificity.

In this article, we highlight the importance of recognizing the typical and atypical presentation patterns of sarcoidosis on CXR and CT, as well as the relevance of thoracic images as key elements in the diagnostic algorithm of this pathology. We also describe the usefulness of magnetic resonance (MR) imaging as an additional method for diagnosis in cases of cardiac involvement and the role of positron emission tomography (PET-CT) in therapeutic follow-up.
Su diagnóstico se basa en la presencia de un cuadro clínico compatible con hallazgos imagenológicos específicos encontrados en la radiografía y tomografía computada de tórax. Posteriormente, esos hallazgos deben ser confirmados por histopatología para llegar a un diagnóstico certero. En casos específicos, la resonancia magnética (RM) se utiliza como método diagnóstico complementario y la tomografía por emisión de positrones (PET-CT) para seguimiento terapéutico.

\section{Historia}

La descripción inicial de la sarcoidosis fue desarrollada por Jonathan Hutchinson que, en 1877, reportó el caso de un paciente con múltiples lesiones cutáneas sobreelevadas, amarronadas y en parches. Posteriormente, en 1899, Caesar Boeck presentó un caso de lesiones cutáneas parecido al reportado por Hutchinson y las describió como "múltiples lesiones sarcoideas benignas de la piel," empleando el término "sarcoideas" debido a que, si bien dichas lesiones se parecían a un sarcoma, eran benignas.

En 1934 Jorgen Schaumann interpretó que esa enfermedad correspondía a una variante de la tuberculosis, por lo cual la denominó linfogranulomatosis benigna. ${ }^{1}$

En 1939, se determinó la relación de hipercalcemia e hipercalciuria con la sarcoidosis y, más tarde, Louis Siltzbach desarrolló una prueba en la que se usaba una suspensión esplénica conocida como la prueba de KveimSiltzbach. ${ }^{2}$ En 1946, Sven Löfgren describió el síndrome de Löfgren, caracterizado por eritema nodoso, adenomegalias hiliares bilaterales, fiebre y poliartritis. ${ }^{3,4}$

\section{Epidemiología}

La prevalencia de sarcoidosis varía de menos de 1 a 40 casos por cada 100.000 personas. Aunque puede presentarse a cualquier edad, afecta generalmente a adultos menores de 40 años y tiene una mayor incidencia en la tercera década de la vida, con una leve dominancia entre las mujeres y en las poblaciones afroamericanas, suecas y danesas. ${ }^{5}$ La mortalidad varía entre el 1 y $8 \%$ y se debe a falla respiratoria por fibrosis pulmonar irreversible y extensa, compromiso cardíaco o neurológico. ${ }^{6}$

En Latinoamérica hay una baja incidencia debido, tal vez, a que esta enfermedad es subdiagnosticada como consecuencia de la dificultad que plantea el diagnóstico diferencial con trastornos similares de mayor prevalencia como tuberculosis, infecciones fúngicas y lepra. ${ }^{7}$

El compromiso intratorácico de la sarcoidosis está presente en el $97 \%$ de los pacientes. El $87 \%$ de los casos presenta adenomegalias intratorácicas y $50 \%$ infiltrados pulmonares. Las manifestaciones extratorácicas más comunes son el rash cutáneo (18\% de los casos), seguido por artralgias (12\%), compromiso oftalmológico (7\%), hepático (6\%), esplénico (4\%), renal (3\%), neurológico (3\%), ganglionar extratorácico (3\%), exocrino (2\%), del tracto respiratorio superior (2\%) y cardíaco (1\%). La afectación ósea es la manifestación extratorácica menos frecuente. ${ }^{8}$

\section{Cuadro clínico}

La sarcoidosis se manifiesta típicamente en mujeres entre los 20 y los 40 años, mientras que en pacientes mayores de 50 años puede presentarse de forma atípica. Si bien hasta el $50 \%$ de los pacientes son asintomáticos, es usual la presencia de síntomas respiratorios como tos, disnea e hiperreactividad bronquial asociados a fatiga, pérdida de peso, sudoración nocturna y eritema nodoso. ${ }^{9}$ Las pruebas de función pulmonar evidencian un defecto ventilatorio restrictivo con disminución de los volúmenes pulmonares y de la capacidad de difusión de monóxido de carbono. 


\section{Etiología}

La causa de la sarcoidosis es desconocida. Sin embargo, se ha atribuido su origen a una respuesta inflamatoria en el sistema inmune en un huésped genéticamente susceptible, desencadenada por agentes ambientales aún no identificados, y en la que intervienen macrófagos alveolares activados y células $\mathrm{T}$. Se ha reportado una mayor incidencia de esa enfermedad en los bomberos que realizaron tareas de rescate en el atentado a las torres gemelas en Nueva York en el año 2001. ${ }^{10}$

\section{Diagnóstico}

El diagnóstico presuntivo se establece en base a hallazgos clínicos e imagenológicos, mientras que el diagnóstico de certeza se basa en la identificación histológica de granulomas no caseificantes en uno o más órganos, en ausencia de otras etiologías.

\section{Diagnóstico Imagenológico}

La radiografía ( $\mathrm{Rx}$ ) y tomografía computada (TC) son los métodos imagenológicos más comúnmente usados para detectar la afectación pulmonar, así como el compromiso ganglionar y bronquial. La RM se utiliza como método complementario en casos con sospecha de afectación cardíaca y el seguimiento con PET-TC para casos puntuales.

\section{Hallazgos Radiográficos}

Hasta en el $85 \%$ de los pacientes se observan adenomegalias en la Rx de tórax. El compromiso más frecuente es hiliar bilateral y paratraqueal derecho (-Fig. 1). También pueden encontrarse adenomegalias en el espacio paratraqueal izquierdo $\mathrm{y}$ en la ventana aortopulmonar, pero su detección es más difícil con ese método. ${ }^{11}$

El compromiso del parénquima pulmonar es mayor a nivel de los lóbulos superiores y se distribuye predominantemente en el espacio intersticial peribroncovascular, subpleural y, en menor medida, en los septos interlobulillares. Esa distribución se manifiesta en la Rx de tórax como patrón nodular, reticular, retículo-nodular y miliar con tendencia a la coalescencia y, menos frecuentemente, puede dar lugar a la formación de masas o nódulos solitarios. ${ }^{12}$

Como hallazgos infrecuentes se pueden observar cavitaciones de las lesiones parenquimatosas sólidas, derrame y engrosamiento pleural, así como compromiso de la vía aérea, evidenciado por estenosis traqueal, bronquiectasias, compresión extrínseca por ganglios linfáticos y obstrucción bronquial secundaria a granulomas, cicatrización y fibrosis. ${ }^{13}$

Existe un sistema de clasificación de la sarcoidosis desarrollado hace más de 40 años por Siltzbach basado en los hallazgos radiológicos de tórax que presenta un gran valor pronóstico para la enfermedad. ${ }^{14}$ Está definido por cinco estadios (-Fig. 2):

- Estadio 0: Sin alteraciones en la radiografía de tórax (de $5 \%$ a $10 \%$ de los casos).

- Estadio I: Adenomegalias hiliares o mediastinales sin evidencia de enfermedad pulmonar (50\%).

- Estadio II: Adenomegalias hiliares o mediastinales con enfermedad pulmonar visible (de 25\% a 30\%).

- Estadio III: Enfermedad pulmonar difusa sin adenomegalias (de $10 \%$ a $12 \%$ ).

- Estadio IV: Fibrosis pulmonar (5\%).
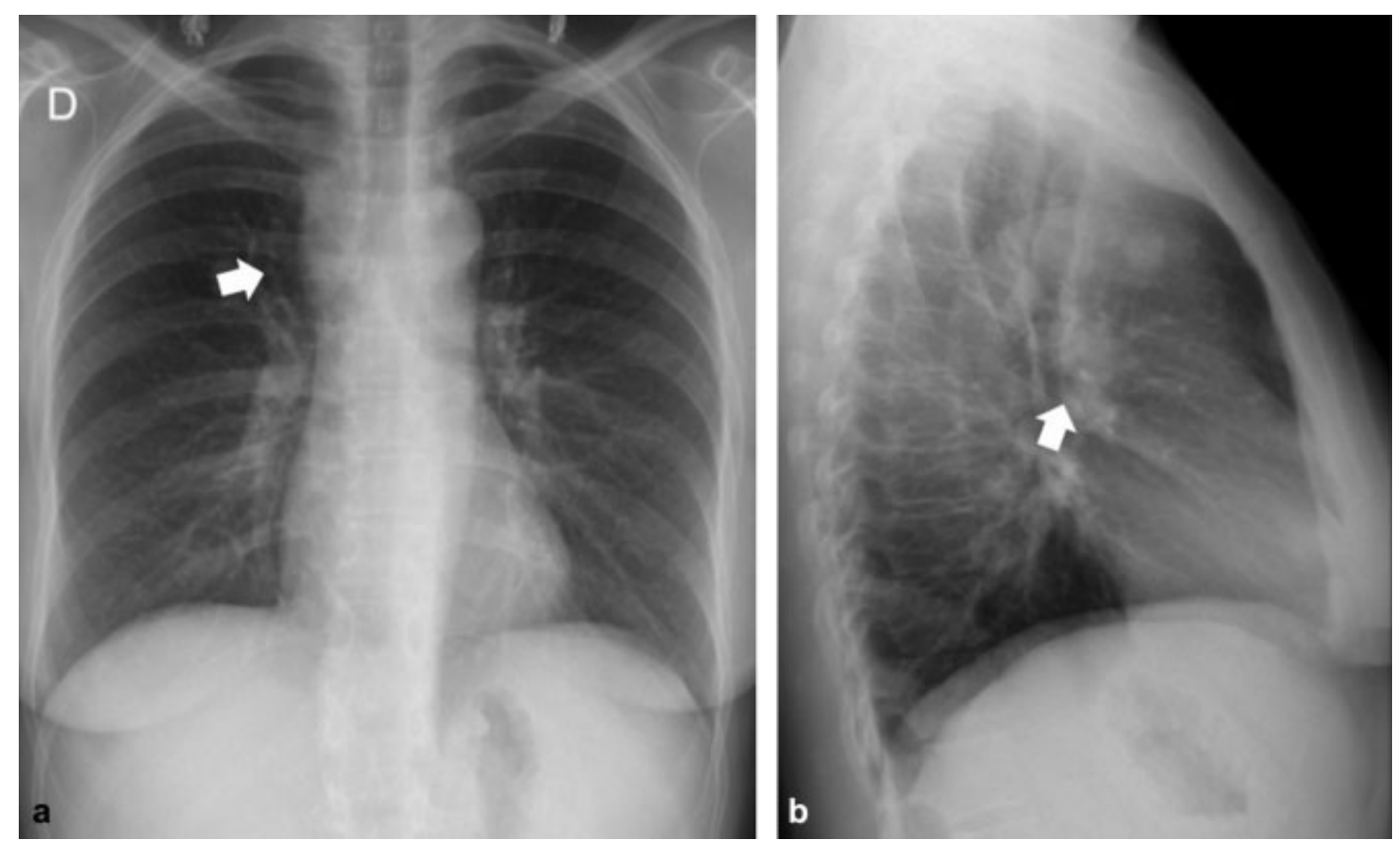

Fig. 1 Paciente femenina de 42 años con diagnóstico de sarcoidosis en estadio I. Rx de frente (a) y perfil (b) que muestran adenomegalias paratraqueales derechas (flechas), como hallazgo frecuente de compromiso mediastinal. 

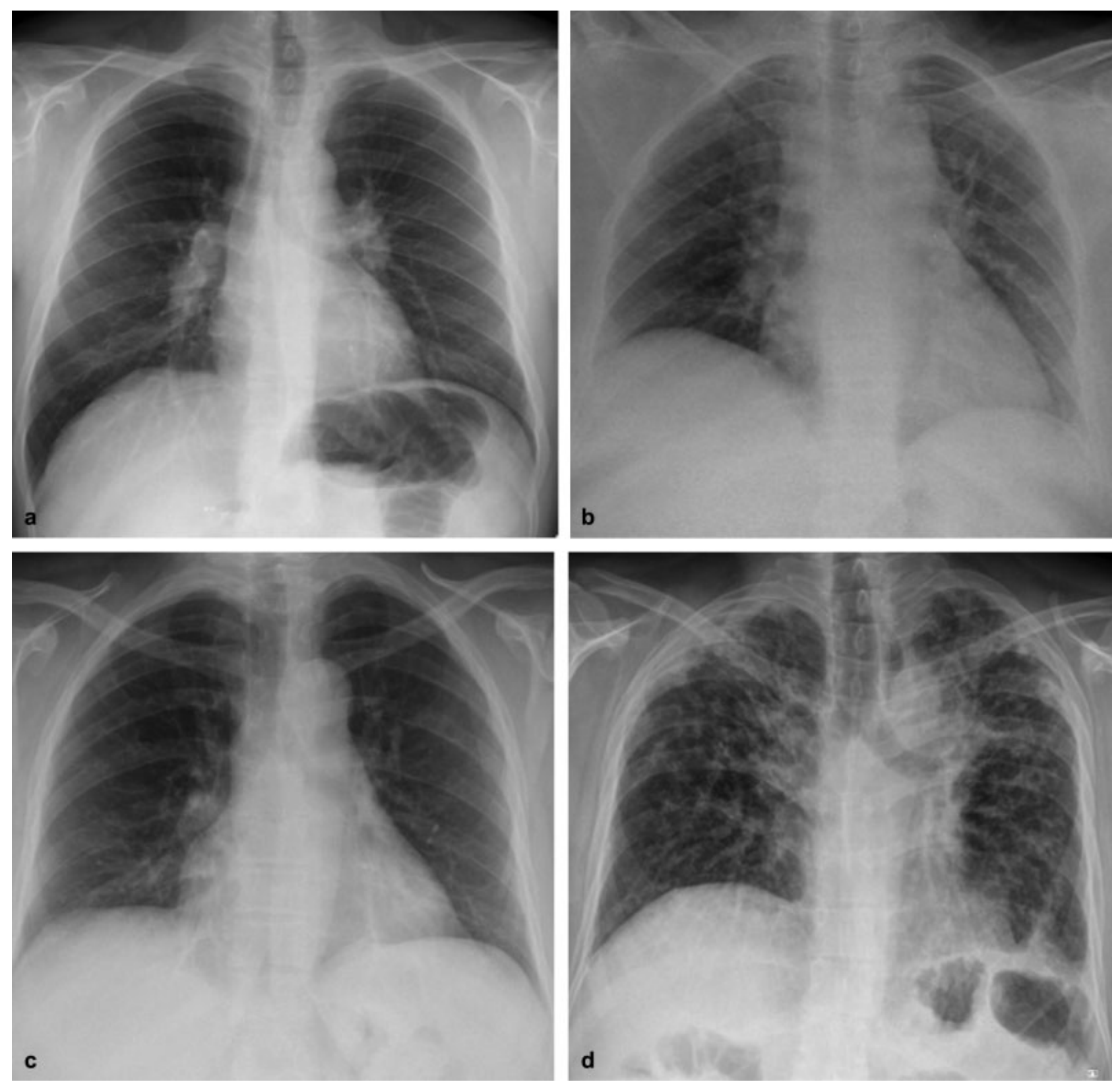

Fig. 2 Estadios de la sarcoidosis. Estadio I (a) adenomegalias mediastinales. Estadio II (b) adenomegalias asociadas a infiltrados pulmonares. Estadio III (c) infiltrados pulmonares sin adenomegalias. Estadio IV (d) se evidencian signos de fibrosis.

\section{Hallazgos Tomográficos}

La TC de tórax multislice ha demostrado ser superior a la tomografía helicoidal en pacientes con sarcoidosis pulmonar, permitiendo la detección temprana de alteraciones parenquimatosas.

\section{Hallazgos Típicos}

El patrón nodulillar de distribución perilinfática es el hallazgo pulmonar más frecuente. Se evidencia entre el 75 y el $90 \%$ de los casos, y se caracteriza por la presencia de nodulillos bilaterales y simétricos, de 2 a $4 \mathrm{~mm}$ de diámetro, con bordes bien definidos, a predominio del lóbulo medio y lóbulos superiores, con tendencia a la coalescencia. Esos nodulillos se encuentran principalmente en el intersticio peribroncovascular, subpleural y, con menor frecuencia, en los septos interlobulillares (-Fig. 3). En estadios avanzados de la enfermedad es habitual encontrar alteración de la arquitectura pulmonar con fibrosis, quistes, bullas y bronquiectasias por tracción (-Fig. 4). ${ }^{9}$

A nivel mediastinal, el patrón más común es el aumento de tamaño de los ganglios paratraqueales derechos (75\% de los casos) e hiliares bilaterales, los cuales son de bordes bien definidos (95\%) (-Fig. 3). Este hallazgo también puede encontrarse en otras etiologías, como enfermedades infecciosas (fúngicas o micobacterianas) o neoplásicas (linfoma o secundarismo). ${ }^{9}$ Los ganglios prevasculares, paratraqueales izquierdos, subcarinales y en ventana aortopulmonar, se encuentran en el $50 \%$ de los casos.

Se ha descrito una nueva morfología de los ganglios linfáticos en TC de tórax con contraste, denominada "en 

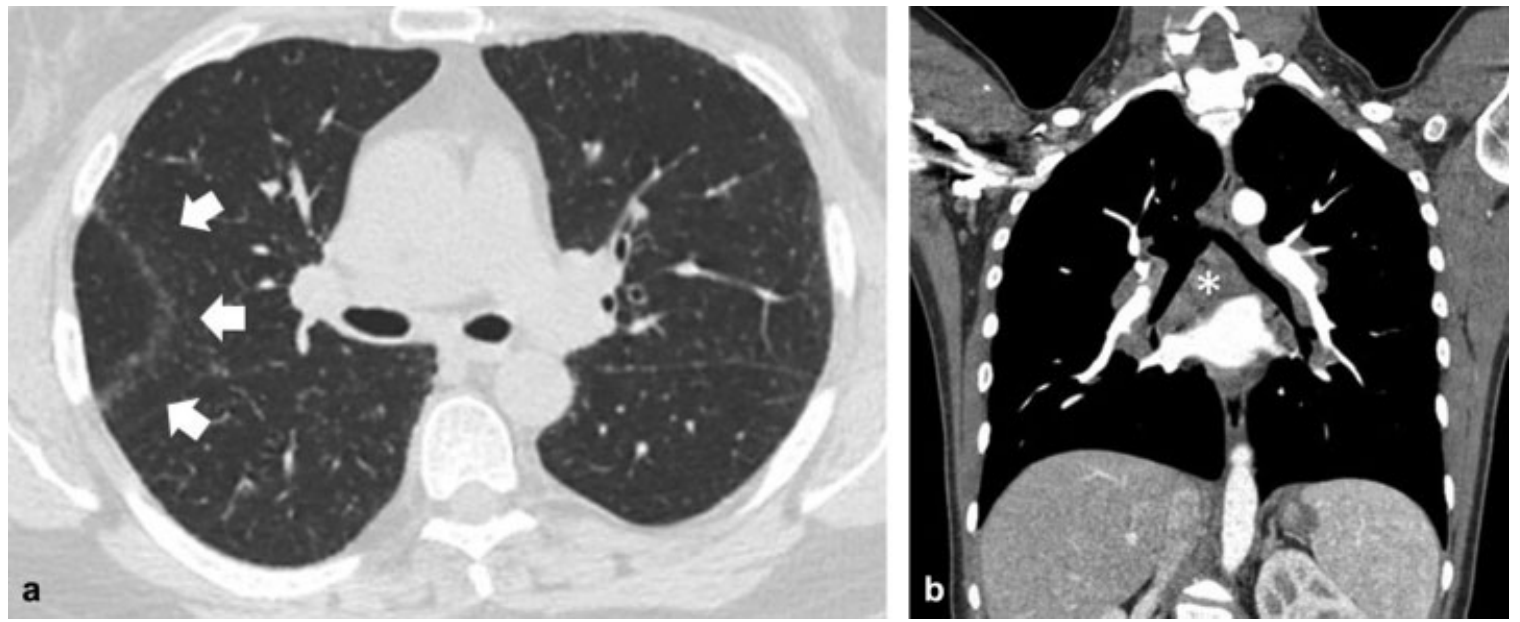

Fig. 3 Hallazgos típicos en sarcoidosis. TC de tórax en axial (a) que muestra múltiples nodulillos de distribución difusa a predominio subpleural y cisural derecho (flechas blancas). TC de tórax con contraste en reconstrucción coronal (b) donde se evidencian múltiples ganglios hiliares bilaterales y mediastinales $\left({ }^{*}\right)$ con engrosamiento del intersticio peribroncovascular.
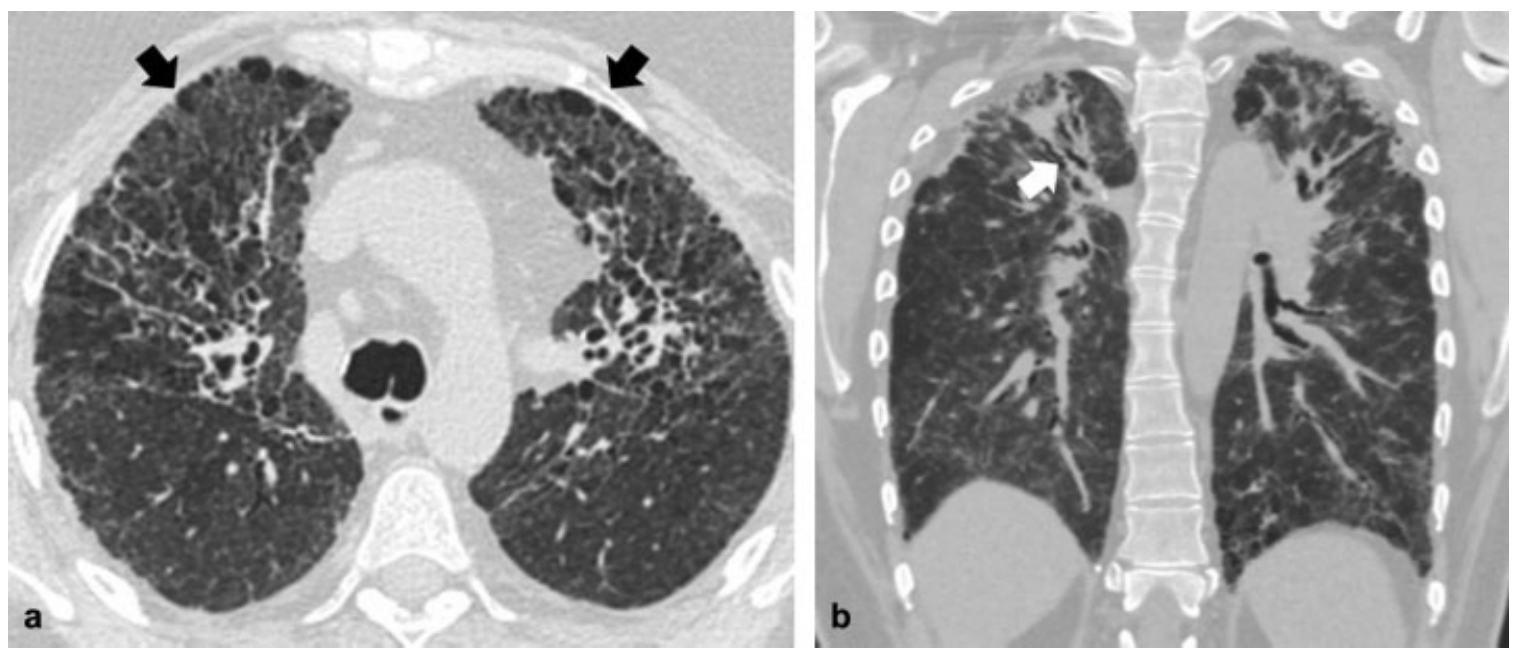

Fig. 4 Fibrosis pulmonar en sarcoidosis. TC de tórax (a y b) donde se visualizan bronquiectasias por tracción (flecha blanca) asociadas a bullas subpleurales (flechas negras). Nótese la afectación predominantemente de ambos lóbulos superiores.

racimo de perlas negras," donde se observan nodulillos hipodensos de 1 a $2 \mathrm{~mm}$ distribuidos uniformemente dentro de un ganglio linfático, que representan granulomas no caseificantes intraganglionares. ${ }^{15}$

\section{Hallazgos atípicos}

Los hallazgos tomográficos son atípicos entre el 25 y el $30 \%$ de los casos e incluyen adenomegalias unilaterales o asimétricas, cavitaciones, consolidaciones, opacidades en vidrio esmerilado, anormalidades de la vía aérea y derrame pleural, entre otros. ${ }^{16}$

Los nódulos y masas pulmonares están presentes entre el 15 y el $25 \%$ de los casos y se presentan como múltiples opacidades parenquimatosas bilaterales, mal definidas e irregulares que se originan por la coalescencia de granulomas intersticiales, miden entre $1 \mathrm{y} 4 \mathrm{~cm}$ y se ubican generalmente a nivel perihiliar o periférico (-Fig. 5). En algunos casos se pueden asociar a broncograma aéreo y cavitaciones (menos del 3\% de los casos) (- Fig. 6). Las masas rodeadas por pequeños nódulos satélites configuran el signo de la "galaxia sarcoidea" (-Fig. 7) que, si bien es un signo característico, también puede encontrarse en otras enfermedades granulomatosas o neoplásicas. ${ }^{17}$

Las opacidades parcheadas en vidrio esmerilado se observan en el $40 \%$ de los pacientes, debido a la compresión extrínseca de la vía aérea generada por los granulomas, y usualmente presentan bordes mal definidos y broncograma aéreo. Dicho patrón también se puede observar en el adenocarcinoma, linfoma, neumonías, neumoconiosis y neumonía organizativa. ${ }^{18}$

Las consolidaciones del espacio aéreo se presentan entre el 10 y el $20 \%$ de los pacientes. Generalmente son bilaterales y simétricas, de bordes mal definidos, y algunas pueden presentar broncograma aéreo. Pueden simular neumonías, tuberculosis o neumonía organizativa.

La panalización en sarcoidosis se distribuye predominantemente en las zonas subpleurales del lóbulo 


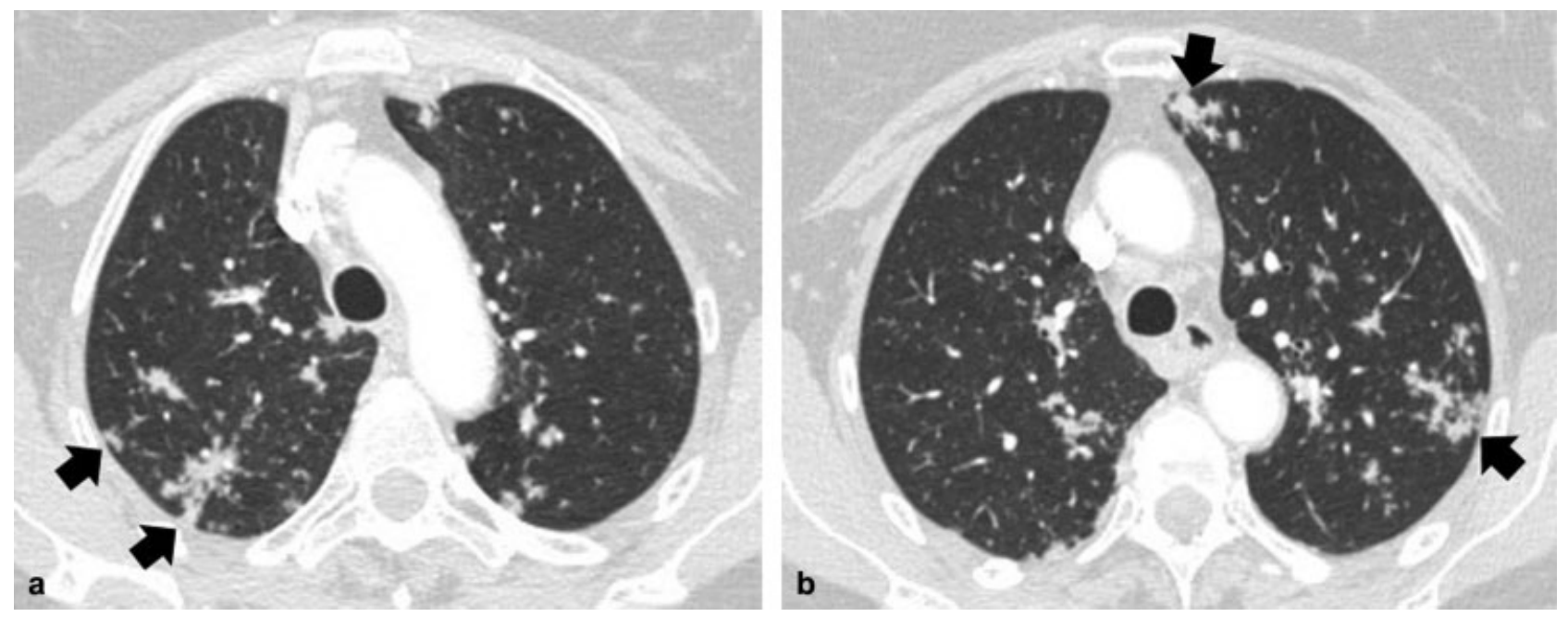

Fig. 5 Mujer de 32 años de edad con diagnóstico de sarcoidosis. TC de tórax (a y b) donde se observan opacidades irregulares de distribución difusa y bilateral (flechas negras) como hallazgo atípico de la enfermedad.
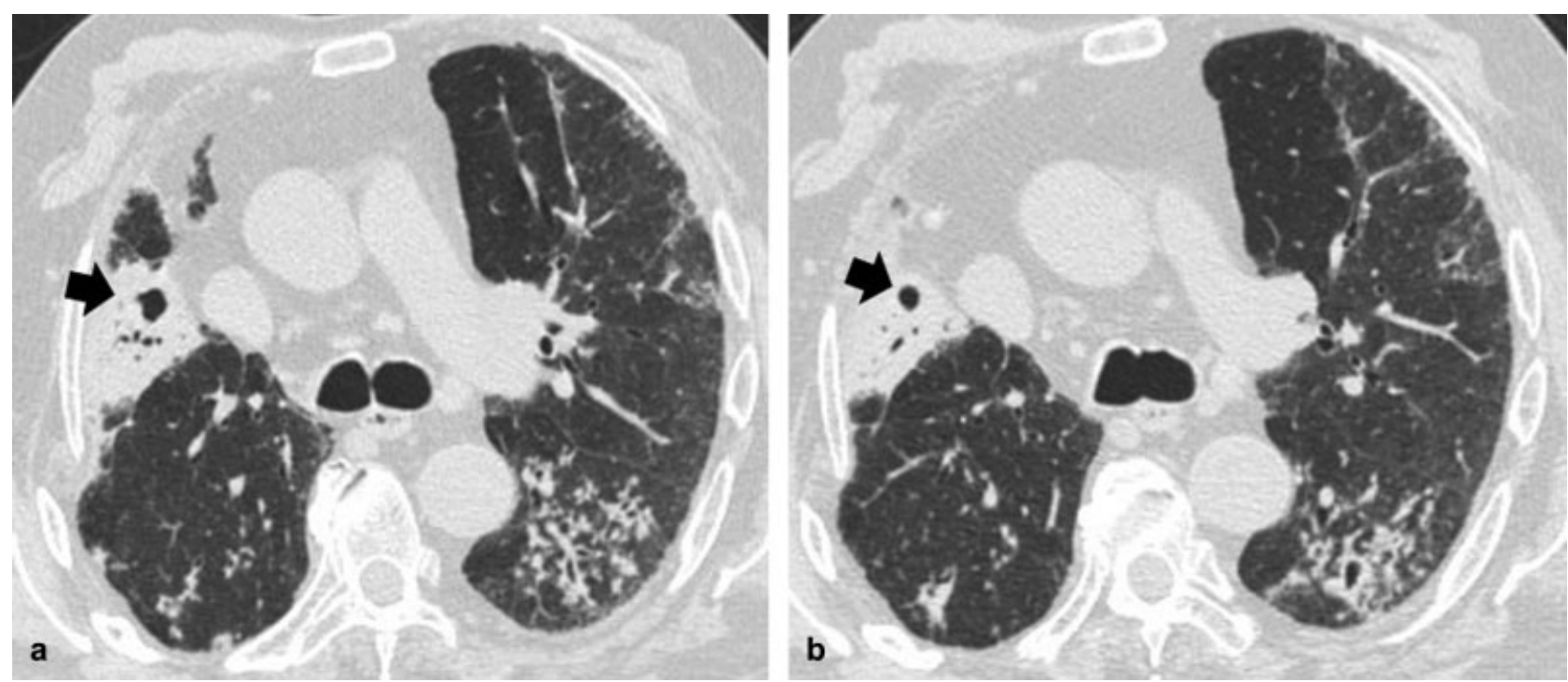

Fig. 6 Paciente femenina de 45 años con diagnóstico conocido de sarcoidosis. Se evidencia una masa cavitada (flecha negra en a y b) como hallazgo atípico de la enfermedad.
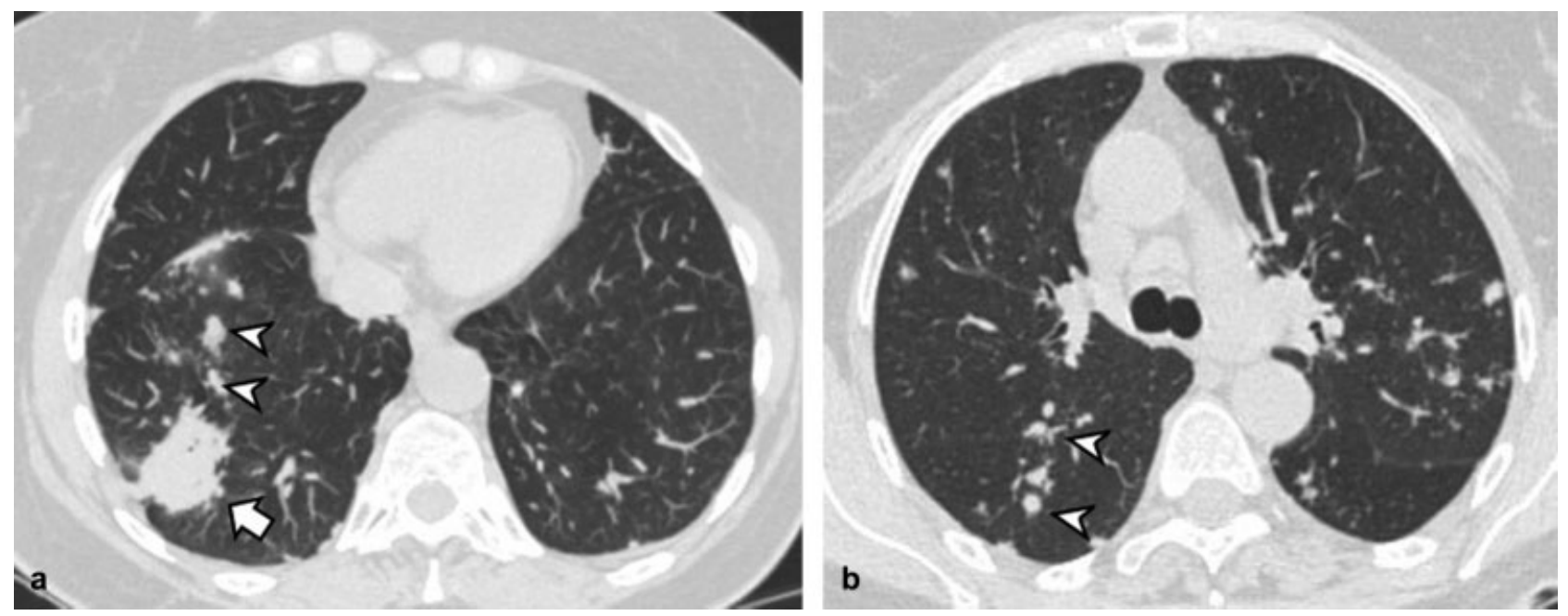

Fig. 7 TC de tórax en mujer de 50 años con diagnóstico de eritema nodoso. Se observa una masa (flecha gruesa en a) y nódulos periféricos (cabezas de flecha en a y b) configurando el signo de la "galaxia sarcoidea." El diagnóstico de sarcoidosis fue confirmado por histolopatología de la lesión. 

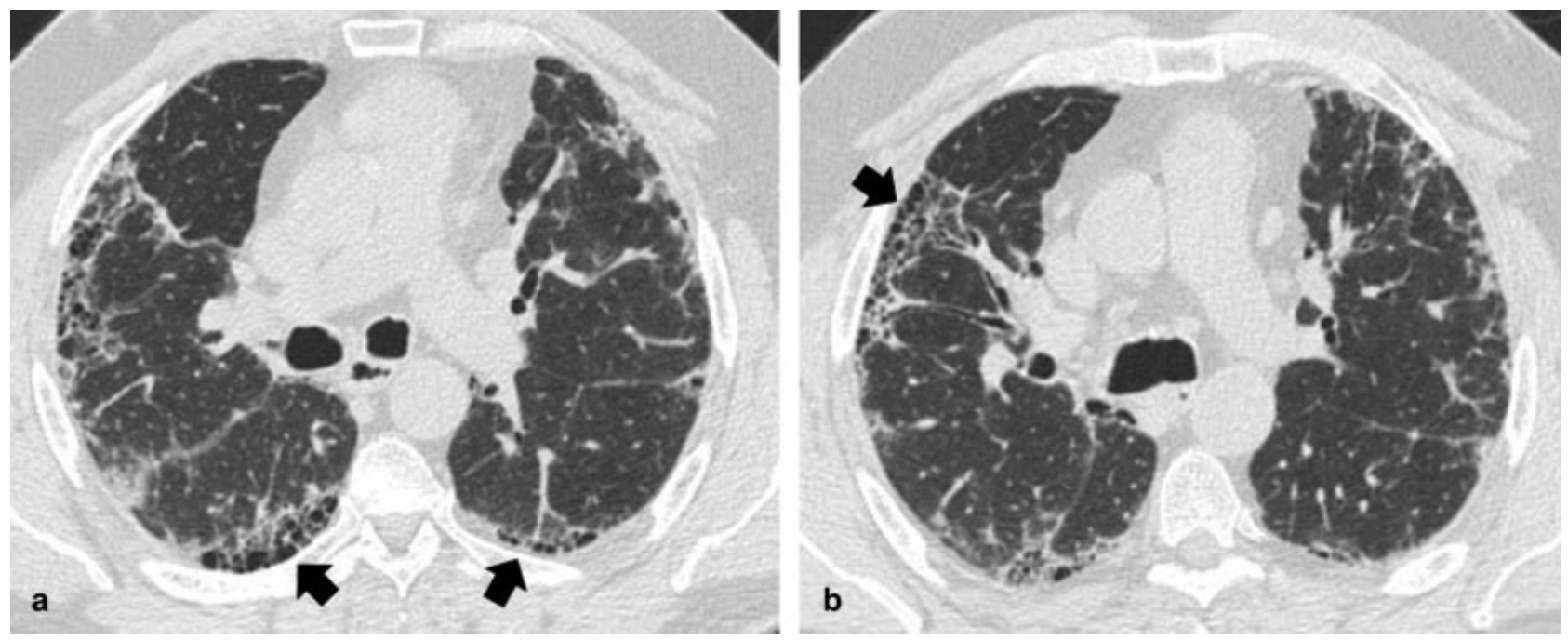

Fig. 8 Paciente masculino de 49 años con fibrosis pulmonar y patrón de panalización como hallazgo en sarcoidosis avanzada que compromete predominantemente los lóbulos superiores (flechas negras en a y b).

medio y lóbulos superiores (-Fig. 8), mientras que las bases pulmonares generalmente están respetadas. En caso de compromiso basal, el patrón puede confundirse con fibrosis pulmonar idiopática.

La cavitación de las lesiones parenquimatosas se puede observar hasta en el $10 \%$ de los pacientes con enfermedad en estadio avanzado, y suelen estar relacionadas a bullas, blebs o quistes preexistentes. ${ }^{19}$

El compromiso pleural es poco frecuente y se presenta como derrame pleural (-Fig. 9), hemotórax, quilotórax, neumotórax, engrosamiento y calcificaciones pleurales. ${ }^{20}$

Es raro encontrar un patrón miliar en sarcoidosis (menos del $1 \%$, y en esos casos, se deben tener en cuenta otros diagnósticos diferenciales como tuberculosis, metástasis, varicela, histoplasmosis, neumoconiosis e histiocitosis de células de Langerhans. ${ }^{21}$

La afectación de la vía aérea se manifiesta por engrosamiento de la pared bronquial de aspecto nodular y obstrucción de los bronquios distales por granulomas.
Además puede provocar atelectasias y anormalidades traqueobronquiales, como por ejemplo estenosis endobronquial, compresión extrínseca, distorsión de los bronquios, patrón en mosaico y atrapamiento aéreo $\left(-\right.$ Fig. 10). ${ }^{22}$

Los hallazgos atípicos mediastinales se caracterizan por una afectación ganglionar asimétrica o en localizaciones inusuales como lo son la cadena paravertebral, mamaria interna y de los ganglios de la región retrocrural. En algunos casos, dichos ganglios pueden presentar calcificaciones amorfas, punteadas, en "palomita de maíz" o "cáscara de huevo" (-Fig. 9).

\section{Hallazgos en PET-TC}

Es frecuente encontrar lesiones parenquimatosas $\mathrm{y}$ conglomerados adenomegálicos mediastinales hipermetabólicos en PET-TC (-Fig. 11), pero tanto la intensidad como el patrón de captación de la ${ }^{18}$ F-FDG son inespecíficos y generalmente no son útiles para el
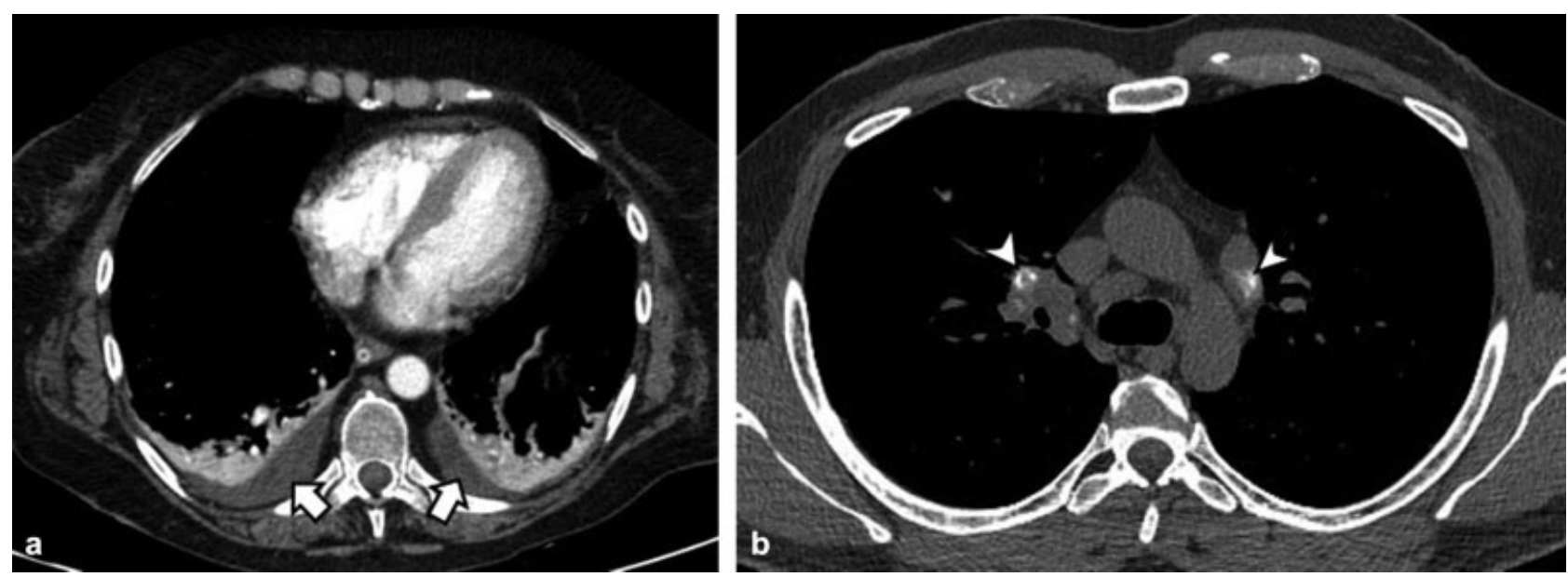

Fig. 9 Derrame pleural en un paciente masculino de 54 años con tos y disnea. TC de tórax (a) que muestra el derrame pleural bilateral (flechas blancas). A nivel del mediastino (b) se observan imágenes ganglionares calcificadas (cabezas de flechas blancas). La biopsia por mediastinoscopia confirmó el diagnóstico de sarcoidosis. 

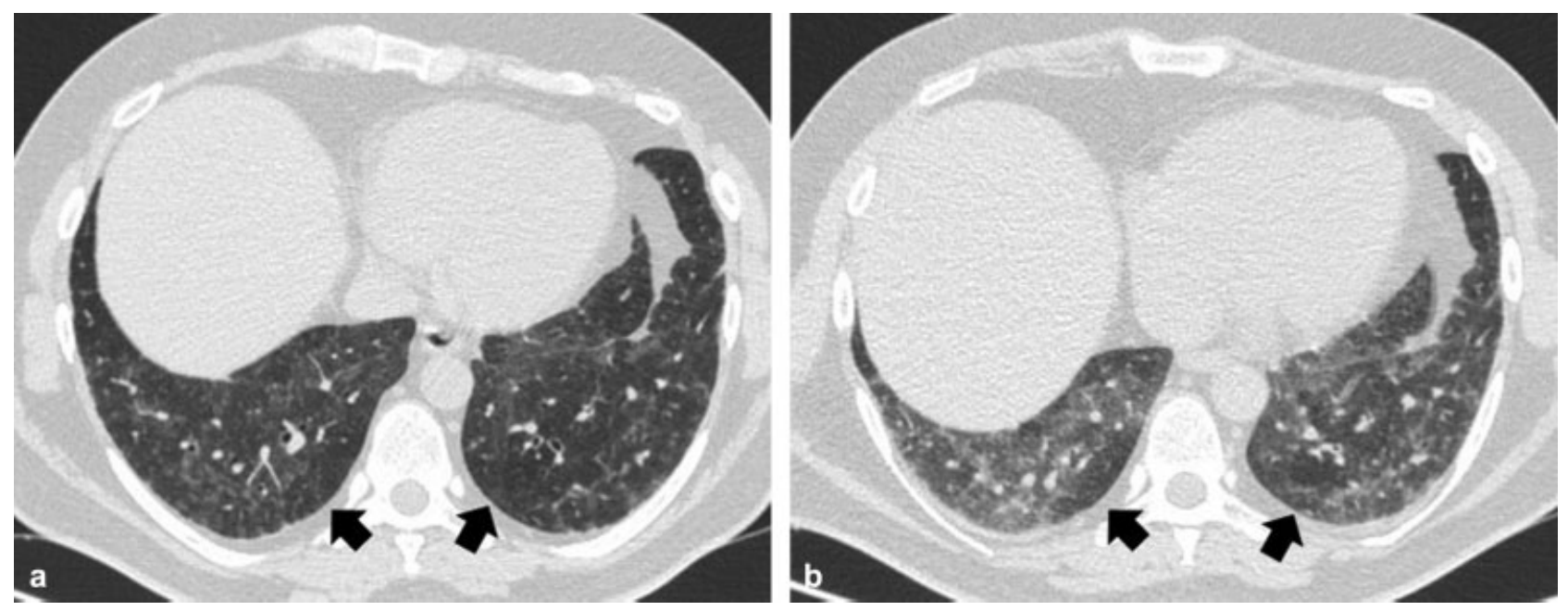

Fig. 10 TC de tórax en inspiración (a) y espiración (b) donde se observan áreas de atrapamiento aéreo (flechas negras) en un paciente masculino con diagnóstico conocido de sarcoidosis.

diagnóstico inicial ni para el diagnóstico diferencial con otras patologías inflamatorias, infecciosas o malignas, ya que la captación puede estar aumentada en todas ellas. ${ }^{23-26}$

Este método es una herramienta útil en el seguimiento terapéutico debido a que la captación del radiofármaco se encuentra aumentada en la enfermedad activa y puede disminuir con el tratamiento, inclusive este método ha demostrado ser más sensible que ciertos parámetros de laboratorio como los niveles de interleucina-2 (IL-2) y enzima convertidora de angiotensina (ECA) en la medición de la actividad inflamatoria en sarcoidosis activa. ${ }^{23,27}$

Si bien el incremento en la captación de la ${ }^{18} \mathrm{~F}$-FDG no es específico para sarcoidosis, los patrones característicos de lesiones pulmonares y adenomegalias, asociadas a un metabolismo aumentado pueden ser suficientes para sugerir el diagnóstico de esa patología ( - Figs. 11 y 12). ${ }^{25}$

Otra utilidad del método es guiar el sitio apropiado para la toma de biopsia, la estimación de la actividad inflamatoria y la extensión de la enfermedad en pacientes con síntomas persistentes atípicos y en los que se sospecha compromiso extrapulmonar. $^{28}$

En medicina nuclear el ${ }^{67} \mathrm{Galio}$ fue empleado inicialmente para el seguimiento de la enfermedad con compromiso cardíaco, pero últimamente ha sido reemplazado por PETTC debido a su mayor sensibilidad. ${ }^{29,30}$

\section{Hallazgos en RM}

Aunque es poco frecuente encontrar afectación cardíaca (entre el $5 \%$ y el $10 \%$ de los casos), la muerte súbita puede ser la única forma de presentación clínica y, por lo tanto, es importante su diagnóstico precoz.

La RM cardíaca con gadolinio es el estudio imagenológico de elección por su alta sensibilidad en la detección de la enfermedad. En la fase inflamatoria se observa, en la pared del ventrículo izquierdo y el septo interventricular, hiperintensidad de señal en secuencia ponderada en T2 con realce tardío de aspecto nodular y parcheado. En las fases inactivas de la enfermedad se evidencian áreas hipointensas en todas las secuencias. ${ }^{31}$ Además, se pueden encontrar adenomegalias mediastinales, derrame pericárdico, engrosamiento miocárdico y pericárdico y anormalidades en la contracción cardíaca.

\section{Hallazgos histológicos}

La confirmación histológica es fundamental en el diagnóstico definitivo de sarcoidosis junto a un cuadro clínico e imágenes diagnósticas compatibles. Histológicamente se observan granulomas no caseificantes formados por un núcleo central de histiocitos, células epitelioides y células gigantes multinucleadas, rodeados periféricamente por linfocitos, células plasmáticas, fibroblastos y colágeno. Esos granulomas están localizados en el intersticio y algunos pueden progresar a fibrosis en estadios avanzados. ${ }^{32,33}$

\section{Pronóstico}

La gran mayoría de los pacientes con sarcoidosis presentan enfermedad estable o remisión completa. Esa última ocurre entre el $60 \%$ y el $90 \%$ y los casos con enfermedad estadio I, entre el $40 \%$ y el $70 \%$ con estadio II, entre el $10 \%$ y el $20 \%$ con estadio III y $0 \%$ con estadio IV. ${ }^{34}$ El resto de los pacientes desarrolla enfermedad crónica caracterizada por fibrosis pulmonar y menos del $8 \%$ mueren por esa patología.

\section{Conclusión}

La Rx y TC de tórax son los métodos imagenológicos más frecuentemente usados en el diagnóstico de la sarcoidosis torácica, siendo la TC más sensible y específica. La presentación típica de la enfermedad incluye nodulillos pulmonares de distribución peribroncovascular y subpleural, junto a adenomegalias paratraqueales derechas e hiliares bilaterales. También puede presentarse de manera atípica, principalmente en pacientes asintomáticos. La RM se puede utilizar como método complementario para evaluar el compromiso cardíaco y PET-TC para el seguimiento terapéutico. 

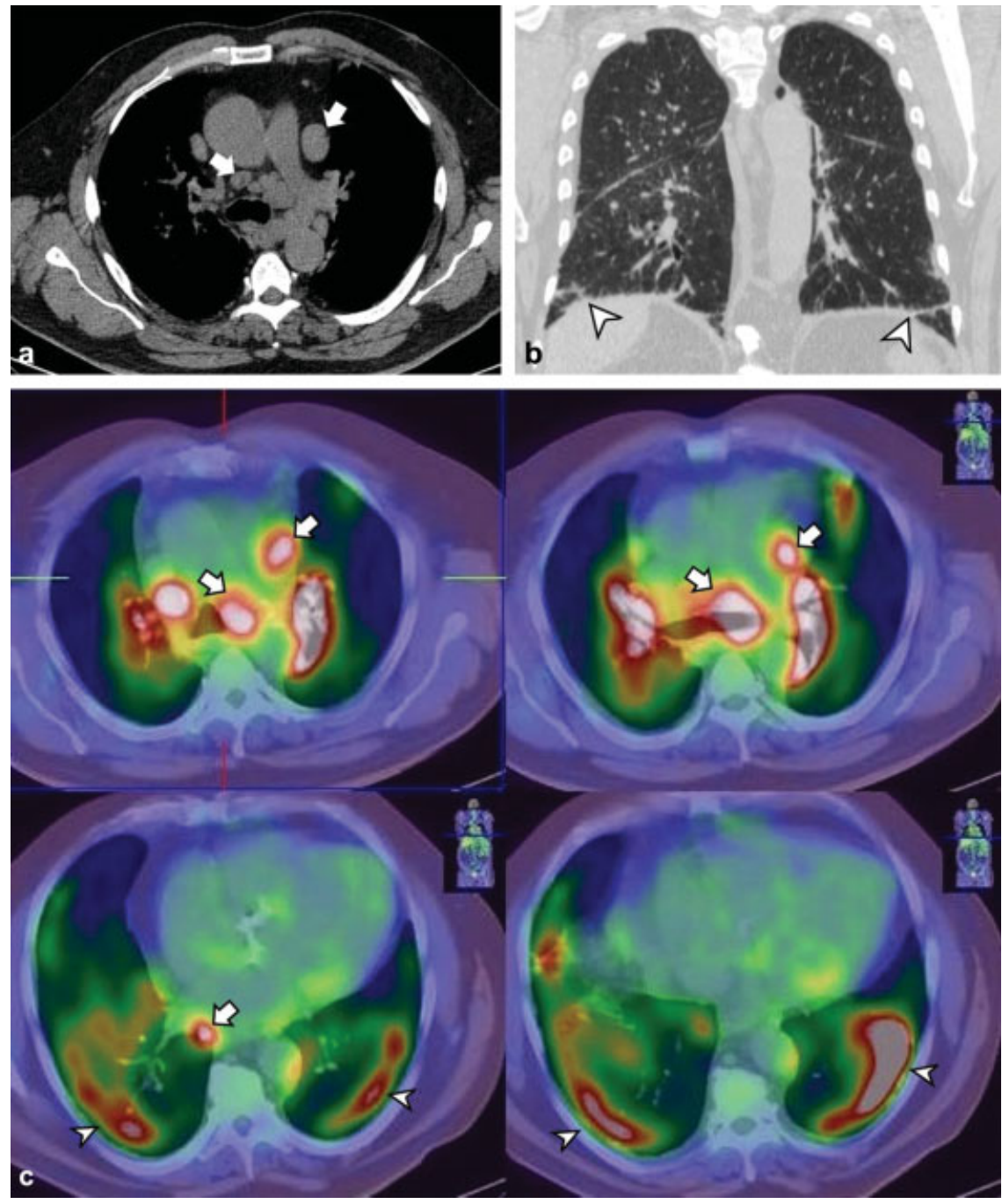

Fig. 11 Mujer de 56 años con disnea. TC de tórax (a) que muestra múltiples adenomegalias mediastinales (flechas blancas) y reconstrucción coronal (b) donde se evidencian engrosamientos pleuroparenquimatosos bibasales (cabezas de flecha). En PET-TC (c) se evidencia la hipercaptación del radiotrazador a nivel de los ganglios mediastinales (flechas blancas) y del parénquima pulmonar (cabezas de flecha). Se realizó videomediastinoscopia y biopsia que confirmó el diagnóstico de sarcoidosis.
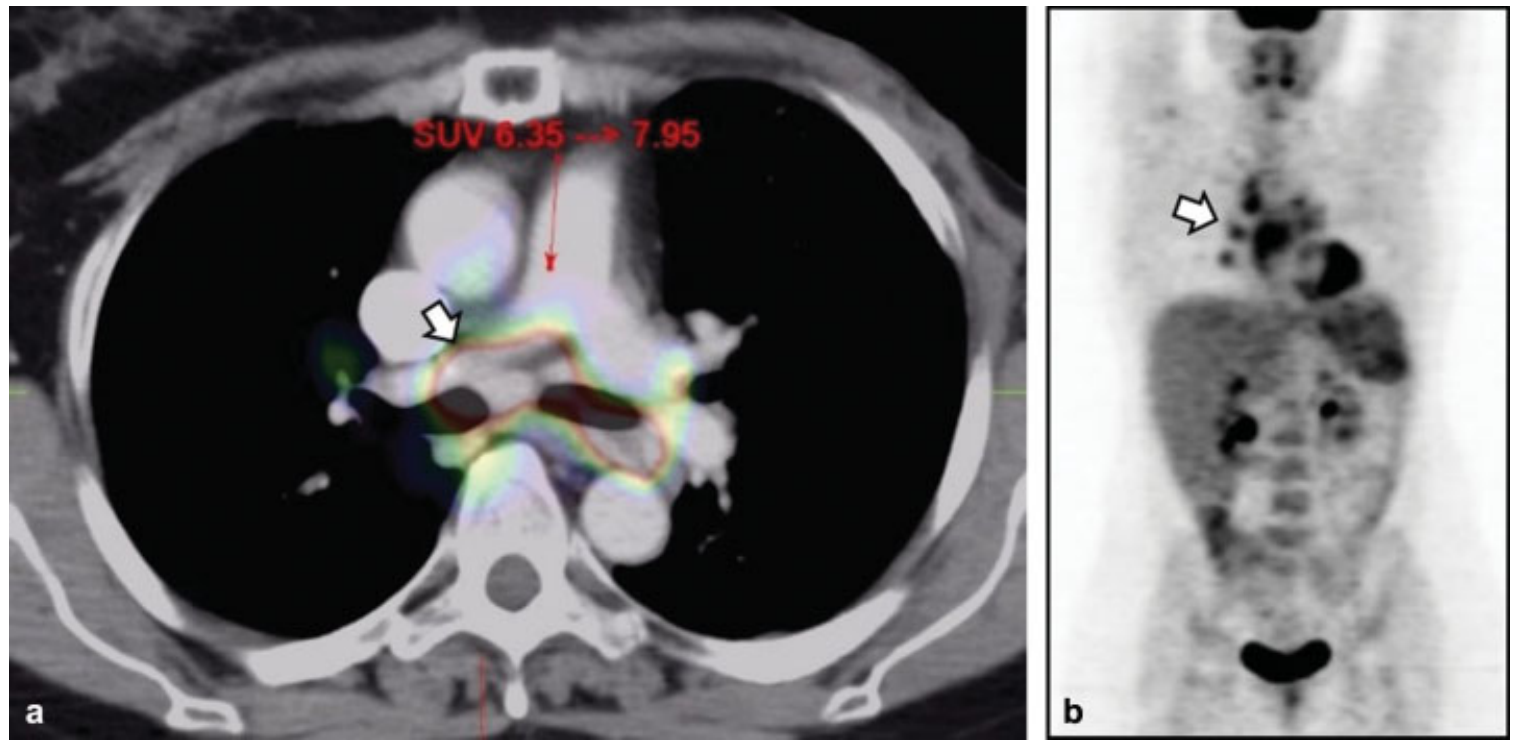

Fig. 12 Paciente de 38 años con tos y pérdida de peso. PET-TC (a) con adenomegalias mediastinales hipermetabólicas con SUV máximo de 7,95. PET volumen MIP (b) donde se observan imágenes ganglionares mediastinales con metabolismo aumentado (flechas blancas). 
Si bien los estudios de imágenes y los datos clínicos son importantes para el diagnóstico presuntivo, se requiere de la confirmación histológica para el diagnóstico definitivo.

\section{Responsabilidades Éticas}

Protección de personas y animales. Los autores declaran que para esta investigación, no se han realizado experimentos en seres humanos ni en animales.

Confidencialidad de los datos. Los autores declaran que han seguido los protocolos de su centro de trabajo sobre la publicación de datos de pacientes.

Derecho a la privacidad y consentimiento informado. Los autores declaran que en este artículo no aparecen datos de pacientes.

Conflicto de Intereses

Los autores declaran no tener ningún conflicto de intereses.

\section{Bibliografía}

1 Hosoda Y, Odaka M. History of sarcoidosis. Semin Respir Crit Care Med 1992;13(05):359-367

2 Siltzbach LE. The Kveim test in sarcoidosis. A study of 750 patients. JAMA 1961;178:476-482

3 Lofgren S. Erythema nodosum: studies on etiology and pathogenesis in 185 adult cases. Acta Med Scand 1946;174:197-395

4 Jiménez S, Muñoz FJ, Cervera R, Xaubet A, Font J. Sarcoidosis. Jano. 2003;1492:1217-1233

5 James DG, Hosoda Y. Epidemiology. En: James DG, ed. Sarcoidosis and other granulomatous disorders. Nueva York: Marcel-Dekker; 1994:729-743

6 Swigris JJ, Olson AL, Huie TJ, et al. Sarcoidosis-related mortality in the United States from 1988 to 2007. Am J Respir Crit Care Med 2011;183(11):1524-1530

7 González EL, Vigliano C, Cáneva J. Sarcoidosis. Presentación clínica y pronóstico. Medicina (B Aires) 2010;70(06):499-502

8 Ungprasert P, Carmona EM, Utz JP, Ryu JH, Crowson CS, Matteson EL. Epidemiology of Sarcoidosis 1946-2013: A Population-Based Study. Mayo Clin Proc 2016;91(02):183-188

9 Criado E, Sánchez M, Ramírez J, et al. Pulmonary sarcoidosis: typical and atypical manifestations at high-resolution CT with pathologic correlation. Radiographics 2010;30(06):1567-1586

10 Girvin F, Zeig-Owens R, Gupta D, et al. Radiologic Features of World Trade Center-related Sarcoidosis in Exposed NYC Fire Department Rescue Workers. J Thorac Imaging 2016;31(05):296-303

11 Miller BH, Rosado-de-Christenson ML, McAdams HP, Fishback NF. Thoracic sarcoidosis: radiologic-pathologic correlation. Radiographics 1995;15(02):421-437

12 Bernstein SS, Sussman ML. Thoracic Manifestations of Sarcoidosis. Radiology 1945;44(01):37-43

13 Rockoff SD, Rohatgi PK. Unusual manifestations of thoracic sarcoidosis. AJR Am J Roentgenol 1985;144(03):513-528

14 Hillerdal G, Nöu E, Osterman K, Schmekel B. Sarcoidosis: epidemiology and prognosis. A 15-year European study. Am Rev Respir Dis 1984;130(01):29-32
15 Venkata Ramanan R, Pudhiavan A, Venkataramanan A. The "cluster of black pearls" sign of sarcoid lymphadenopathy: a new sign on thin-section contrast-enhanced multidetector CT. Clin Radiol 2017;72(09):729-736

16 Park HJ, Jung JI, Chung MH, et al. Typical and atypical manifestations of intrathoracic sarcoidosis. Korean J Radiol 2009;10(06):623-631

17 Nakatsu M, Hatabu H, Morikawa K, et al. Large coalescent parenchymal nodules in pulmonary sarcoidosis: "sarcoid galaxy” sign. AJR Am J Roentgenol 2002;178(06):1389-1393

18 Al-Jahdali H, Rajiah P, Koteyar SS, Allen C, Khan AN. Atypical radiological manifestations of thoracic sarcoidosis: A review and pictorial essay. Ann Thorac Med 2013;8(04):186-196

19 Ichikawa Y, Fujimoto K, Shiraishi T, Oizumi K. Primary cavitary sarcoidosis: high-resolution CT findings. AJR Am J Roentgenol 1994;163(03):745

20 Soskel NT, Sharma OP. Pleural involvement in sarcoidosis: case presentation and detailed review of the literature. Semin Respir Crit Care Med 1992;13(06):492-514

21 Wells A. High resolution computed tomography in sarcoidosis: a clinical perspective. Sarcoidosis Vasc Diffuse Lung Dis 1998;15 (02):140-146

22 Udwadia ZF, Pilling JR, Jenkins PF, Harrison BD. Bronchoscopic and bronchographic findings in 12 patients with sarcoidosis and severe or progressive airways obstruction. Thorax 1990;45(04): $272-275$

23 Prabhakar HB, Rabinowitz CB, Gibbons FK, O'Donnell WJ, Shepard JAO, Aquino SL. Imaging features of sarcoidosis on MDCT, FDG PET, and PET/CT. AJR Am J Roentgenol 2008;190 (3, Suppl):S1-S6

24 Lewis PJ, Salama A. Uptake of fluorine-18-fluorodeoxyglucose in sarcoidosis. J Nucl Med 1994;35(10):1647-1649

25 Love C, Tomas MB, Tronco GG, Palestro CJ. FDG PET of infection and inflammation. Radiographics 2005;25(05):1357-1368

26 Shetty A, Carter JD. Sarcoidosis mimicking lymphoma on FDG-PET imaging. Radiol Case Rep 2015;6(02):409

27 Keijsers RG, Verzijlbergen FJ, Oyen WJ, et al. 18F-FDG PET, genotype-corrected ACE and SIL-2R in newly diagnosed sarcoidosis. Eur J Nucl Med Mol Imaging 2009;36(07):1131-1137

28 Serafim-Chrysovalantis K, Evaggelia F, Christoforos E, et al. The role of PET scan in differential diagnosis of extrapulmonary involvement in sarcoidosis. Ann Transl Med 2016;22(04):

29 Shammas RL, Movahed A. Sarcoidosis of the heart. Clin Cardiol 1993;16(06):462-472

30 Nishiyama Y, Yamamoto Y, Fukunaga K, et al. Comparative evaluation of 18F-FDG PET and 67Ga scintigraphy in patients with sarcoidosis. J Nucl Med 2006;47(10):1571-1576

31 García-Gallego F, Bret-Zurita M, García-Quero C, Cuesta-López E. Sarcoidosis cardiaca: diagnóstico por resonancia magnética con contraste. Rev Esp Cardiol 2008;61(07):784-786

32 Silverstein E, Pertschuk LP, Friedland J. Immunofluorescent localization of angiotensin converting enzyme in epithelioid and giant cells of sarcoidosis granulomas. Proc Natl Acad Sci U S A 1979;76(12):6646-6648

33 Okabe T, Ishizuka S, Fujisawa M, Watanabe J, Takaku F. Sarcoid granulomas metabolize 25-hydroxyvitamin D3 in vitro. Biochem Biophys Res Commun 1984;123(02):822-830

34 Siltzbach LE. Sarcoidosis: clinical features and management. Med Clin North Am 1967;51(02):483-502 